\title{
Sanfilippo Syndrome
}

National Cancer Institute

\section{Source}

National Cancer Institute. Sanfilippo Syndrome. NCI Thesaurus. Code C61262.

A rare autosomal recessive lysosomal storage disease affecting the metabolism of mucopolysaccharides. Signs and symptoms include behavioral changes, sleep disorders, mental developmental delays, and seizures. 